\title{
GLOBAL EXISTENCE OF SOLUTIONS TO NAVIER-STOKES EQUATIONS IN CYLINDRICAL DOMAINS
}

Abstract. We prove the existence of global and regular solutions to the Navier-Stokes equations in cylindrical type domains under boundary slip conditions, where coordinates are chosen so that the $x_{3}$-axis is parallel to the axis of the cylinder. Regular solutions have already been obtained on the interval $[0, T]$, where $T>0$ is large, on the assumption that the $L_{2}$-norms of the third component of the force field, of derivatives of the force field, and of the velocity field with respect to the direction of the axis of the cylinder are small. In this paper we continue the solution to all times.

1. Introduction. We consider the following initial-boundary value problem:

$$
\begin{array}{ll}
v_{, t}+v \cdot \nabla v-\operatorname{div} \mathbb{T}(v, p)=f & \text { in } \Omega \times(0, \infty), \\
\operatorname{div} v=0 & \text { in } \Omega \times(0, \infty), \\
v \cdot n=0 & \text { on } S \times(0, \infty), \\
n \cdot \mathbb{T}(v, p) \cdot \tau_{\alpha}=0, \quad \alpha=1,2, & \text { on } S \times(0, \infty), \\
\left.v\right|_{t=0}=v(0) & \text { in } \Omega .
\end{array}
$$

The domain $\Omega$ is an open and bounded subset of $\mathbb{R}^{3}$ of cylindrical type, not axially symmetric but parallel to the $x_{3}$-axis in the Cartesian coordinate system $x=\left(x_{1}, x_{2}, x_{3}\right)$. The velocity field is denoted by $v=v(x, t)=$ $\left(v_{1}(x, t), v_{2}(x, t), v_{3}(x, t)\right) \in \mathbb{R}^{3}$, the external force field is denoted by $f=$ $f(x, t)=\left(f_{1}(x, t), f_{2}(x, t), f_{3}(x, t)\right) \in \mathbb{R}^{3}$, and $p=p(x, t) \in \mathbb{R}^{1}$ is the pressure. We denote by $n$ the unit outward normal vector and by $\tau_{\alpha}, \alpha=1,2$, the tangent vectors to the boundary $S$. Moreover, $\mathbb{T}(v, p)$ is the stress tensor,

2000 Mathematics Subject Classification: 35Q35, 76D03, 76D05.

Key words and phrases: Navier-Stokes equations, global existence of regular solutions, cylindrical type domains, boundary-slip conditions. 
which is equal to $\nu \mathbb{D}(v)-p \mathbb{I}$, where $\mathbb{D}(v)=\nabla v+(\nabla v)^{T}$ is the symmetric dilatation tensor and $\nu>0$ is the constant viscosity coefficient.

The aim of this paper is to show the global in time existence of regular solutions to (1.1). We base on [4], where the existence of a regular solution for large time has been proved by the Leray-Schauder fixed point theorem. Using the existence of the solution on $[0, T]$ we continue it on $\mathbb{R}_{+}$by a recursive procedure employing some cut-off functions. A similar technique has been used in [2]. The main results of the paper are stated in the following theorems.

Theorem 1 (local existence). Let

$\delta_{k}(T):=\left\|f_{, x_{3}}\right\|_{L_{2}(\Omega \times(k T,(k+1) T))}+\left\|f_{3}\right\|_{L_{2}\left(S_{2} \times(k T,(k+1) T)\right)}+\left\|v_{, x_{3}}(k T)\right\|_{L_{2}(\Omega)}$, where $k \in \mathbb{N}$. Assume that

$$
\begin{aligned}
& f \in L_{\infty}\left(k T,(k+1) T ; L_{6 / 5}(\Omega)\right) \cap L_{2}(\Omega \times(k T,(k+1) T)), \\
& f_{3} \in L_{2}\left(S_{2} \times(k T,(k+1) T)\right), \\
& (\operatorname{rot} f)_{3} \in L_{2}\left(k T,(k+1) T ; L_{6 / 5}(\Omega)\right), \\
& f_{x_{3}} \in L_{2}(\Omega \times(k T,(k+1) T)) \cap L_{\sigma}(\Omega \times(k T,(k+1) T)), \\
& v_{x_{3}} \in L_{\sigma}(\Omega \times((k-1) T, k T))
\end{aligned}
$$

and $v(k T) \in H^{1}(\Omega)$. Then, if $\delta_{k}(T)$ is small enough, then there exists a solution to (1.1) such that

$$
\left\|v_{, x_{3}}\right\|_{W_{\sigma}^{2,1}(\Omega \times(k T,(k+1) T))}+\left\|\nabla p_{, x_{3}}\right\|_{L_{\sigma}(\Omega \times(k T,(k+1) T)}<A
$$

and

$$
\|v\|_{W_{2}^{2,1}(\Omega \times(k T,(k+1) T))}+\|\nabla p\|_{L_{2}(\Omega \times(k T,(k+1) T)}<c\left(A^{2}+1\right)
$$

for any $\sigma \in(25 / 8,10 / 3)$. The constant $A$ is chosen for a given $T$ and it satisfies the inequalities

$$
\varphi\left(3 A+D_{k}\right) \delta_{k}(T)+c E_{k} \leq A, \quad c E_{k}<A,
$$

where $\varphi$ is some nonlinear, positive and increasing function, the constant $c$ comes from an imbedding theorem for Sobolev spaces, and the constants $D_{k}$ and $E_{k}$ are given by

$$
\begin{aligned}
D_{k}:= & \|f\|_{L_{\infty}\left(k T,(k+1) T ; L_{6 / 5}(\Omega)\right)}+\left\|f_{3}\right\|_{L_{2}\left(S_{2} \times(k T,(k+1) T)\right)}+\|f\|_{L_{2}(\Omega \times(k T,(k+1) T))} \\
& +\left\|(\operatorname{rot} f)_{3}\right\|_{L_{2}\left(k T,(k+1) T ; L_{6 / 5}(\Omega)\right)}+\left\|f_{, x_{3}}\right\|_{L_{2}(\Omega \times(k T,(k+1) T))}+d_{1}+d_{2}, \\
E_{k}:= & \left\|f_{x_{3}}\right\|_{L_{\sigma}(\Omega \times(k T,(k+1) T))},
\end{aligned}
$$

where $d_{1}$ and $d_{2}$ come from the energy estimates of weak solutions to the problem (1.1) (see Lemma 2.2).

TheOREM 2 (global existence). Under the assumptions of Theorem 1 on external data there exists a global and regular solution to the problem (1.1) 
such that

$$
\left\|v_{, x_{3}}\right\|_{W_{\sigma}^{2,1}(\Omega \times(k T,(k+1) T))}+\left\|\nabla p_{, x_{3}}\right\|_{L_{\sigma}(\Omega \times(k T,(k+1) T))}<A
$$

and

$$
\|v\|_{W_{2}^{2,1}(\Omega \times(k T,(k+1) T))}+\|\nabla p\|_{L_{2}(\Omega \times(k T,(k+1) T))}<c\left(A^{2}+1\right),
$$

where $\sigma$ and $A$ are as in Theorem 1 and the constant $A$ does not depend on $k$.

TheOrem 3 (uniqueness). Any solution to the problem (1.1) which belongs to the space $L_{\infty}\left(k T,(k+1) T ; W_{3}^{1}(\Omega)\right)$ is unique.

The proof of Theorem 1 in the case $k=0$ is presented in [4]. In this paper we will show how to obtain the constant $A$ independent of $k$.

2. Notation and auxiliary results. Let $\Omega^{k T}$ denote $\Omega \times(k T,(k+1) T)$. We introduce the spaces

$$
\begin{aligned}
& V_{2}^{m}\left(\Omega^{k T}\right)=\left\{u:\|u\|_{V_{2}^{m}\left(\Omega^{k T}\right)}=\underset{t \in(k T,(k+1) T)}{\operatorname{ess} \sup \|v\|_{H^{m}}(\Omega)}\right. \\
& \left.+\left(\int_{k T}^{(k+1) T}\|\nabla u\|_{H^{m}(\Omega)}^{2} d t\right)^{1 / 2}<\infty\right\}, \\
& W_{\sigma}^{2,1}\left(\Omega^{k T}\right)=\left\{u:\|v\|_{W_{\sigma}^{2,1}\left(\Omega^{k T}\right)}\right. \\
& \left.=\left(\int_{\Omega^{k T}}\left(|u|^{\sigma}+|D u|^{\sigma}+\left|D^{2} u\right|^{\sigma}+\left|\partial_{t} u\right|^{\sigma}\right) d x d t\right)^{1 / \sigma}<\infty\right\}
\end{aligned}
$$

and

$$
\begin{aligned}
W_{\sigma}^{l}(\Omega)=\left\{u:\|u\|_{W_{\sigma}^{l}(\Omega)}^{\sigma}=\|u\|_{L_{\sigma}(\Omega)}\right. & +\sum_{0 \leq l^{\prime} \leq[l]} \int_{\Omega}\left|D_{x}^{l^{\prime}} u(x)\right|^{\sigma} d x \\
& \left.+\iint_{\Omega} \frac{\left|D_{x}^{[l]} u(x)-D_{x^{\prime}}^{[l]} u\left(x^{\prime}\right)\right|^{\sigma}}{\left|x-x^{\prime}\right|^{3+\sigma(l-[l])}} d x d x^{\prime}<\infty\right\},
\end{aligned}
$$

where $k, m \in \mathbb{N} \cup\{0\}, l$ is any positive real number and $L_{\sigma}$ is the Lebesgue space.

Our approach requires the energy estimates of weak solutions to the problem (1.1). They are obtained by application of the following

LEMMA 2.1 (Korn inequality). Assume that $v \in H^{1}(\Omega)$ satisfies

$$
\begin{aligned}
& \|\mathbb{D}(v)\|_{L_{2}(\Omega)}^{2}<\infty, \\
& \left.v \cdot n\right|_{S}=0, \\
& \operatorname{div} v=0
\end{aligned}
$$


If $\Omega$ is not axially symmetric, then there exists a constant $c_{1}$ such that

$$
\|v\|_{H^{1}(\Omega)}^{2} \leq c_{1}\|\mathbb{D}(v)\|_{L_{2}(\Omega)}^{2} .
$$

The proof can be found in [6, Sec. 1, Lemma 1.2].

Our considerations involve three global quantities:

$$
\begin{aligned}
& a_{1}=\sup _{t}\|f(t)\|_{L_{6 / 5}(\Omega)}, \\
& d_{1}^{2}=\frac{c}{\nu_{1}} a_{1}^{2}+\|v(0)\|_{L_{2}(\Omega)}^{2}, \\
& d_{2}^{2}=\left(\min \left(1, \nu_{2}\right)\right)^{-1} e^{\nu_{1} T}\left(\frac{c}{\nu_{1}} a_{1}^{2}+d_{1}^{2}\right),
\end{aligned}
$$

which do not depend on $k \in \mathbb{N}$ and $\nu / c_{1}=\nu_{1}+\nu_{2}$, where $c_{1}$ is the constant from the Korn inequality (Lemma 2.1).

Finally we can present the energy estimates of weak solutions to the problem (1.1). Then

LEMMA 2.2. Assume that $a_{1}<\infty, v(0) \in L_{2}(\Omega)$ and $T>0$ are given.

$$
\begin{array}{ll}
\|v(t)\|_{L_{2}(\Omega)} \leq d_{1} & \text { for any } t \geq 0, \\
\|v\|_{V_{2}^{0}(\Omega \times(k T, t))} \leq d_{2} & \text { for } t \in(k T,(k+1) T), k \in \mathbb{N} .
\end{array}
$$

For convenience of notation we will use the following quantities that have been introduced in [4]:

$$
\begin{array}{ll}
h=v_{x_{3}}, & q=p_{, x_{3}}, \quad g=f_{, x_{3}}, \quad \chi=(\operatorname{rot} v)_{3}=v_{2, x_{1}}-v_{1, x_{2}}, \\
w=v_{3}, & F_{3}=(\operatorname{rot} f)_{3} .
\end{array}
$$

These functions solve equations and satisfy estimates which we recall in the following lemmas.

LEMMA 2.3. The pair of functions $(h, q)$ is a solution to the problem

$$
\begin{array}{lll} 
& h_{, t}-\operatorname{div} \mathbb{T}(h, q)=-v \cdot \nabla h-h \cdot \nabla v+g & \text { in } \Omega^{T} \equiv \Omega \times(0, T), \\
& \operatorname{div} h=0 & \text { in } \Omega^{T}, \\
(2.1) & h \cdot n=0, \quad n \cdot \mathbb{T}(h, q) \cdot \tau_{\alpha}=0, \quad \alpha=1,2, & \text { on } S_{1}^{T} \equiv S_{1} \times(0, T), \\
& h_{i}=0, \quad i=1,2, \quad h_{3, x_{3}}=0 & \text { on } S_{2}^{T} \equiv S_{2} \times(0, T), \\
& \left.h\right|_{t=0}=h(0) & \text { in } \Omega .
\end{array}
$$

Lemma 2.4. Let $v$ be a weak solution to the problem (1.1). Assume that $h \in L_{\infty}\left(0, T ; L_{3}(\Omega)\right), g \in L_{2}\left(\Omega^{T}\right), f_{3} \in L_{2}\left(S_{2}^{T}\right)$ and $h(0) \in L_{2}(\Omega)$. Then

$$
\|h\|_{V_{2}^{0}\left(\Omega^{t}\right)}^{2} \leq c d_{2}^{2}\|h\|_{L_{\infty}\left(0, t ; L_{3}(\Omega)\right)}^{2}+c\left(\left\|f_{3}\right\|_{L_{2}\left(S_{2}^{t}\right)}^{2}+\|g\|_{L_{2}\left(\Omega^{t}\right)}^{2}+\|h(0)\|_{L_{2}(\Omega)}^{2}\right)
$$

for all $t \leq T$. 
Lemma 2.5. Let $v$ be a weak solution to the problem (1.1). Assume that $\nabla v \in L_{2}\left(0, T ; L_{3}(\Omega)\right), h \in L_{\infty}\left(0, T ; L_{3}(\Omega)\right), g \in L_{2}\left(\Omega^{T}\right), f_{3} \in L_{2}\left(S_{2}^{T}\right)$ and $h(0) \in L_{2}(\Omega)$. Then

$$
\begin{aligned}
&\|h\|_{L_{2}\left(\Omega^{t}\right)} \leq c\left(\|\nabla v\|_{L_{2}\left(0, t ; L_{3}(\Omega)\right)} \exp \left(c\|\nabla v\|_{L_{2}\left(0, t ; L_{3}(\Omega)\right)}^{2}\right)+1\right) \\
& \cdot\left(\left\|f_{3}\right\|_{L_{2}\left(S_{2}^{t}\right)}+\|g\|_{L_{2}\left(\Omega^{t}\right)}+\|h(0)\|_{L_{2}(\Omega)}\right)
\end{aligned}
$$

for all $t \leq T$.

Lemma 2.6. Let $q$ and $f_{3}$ be given. Then $w$ is a solution to the problem

$$
\begin{array}{ll}
w_{, t}+v \cdot \nabla w-\nu \Delta w=q+f_{3} & \text { in } \Omega^{T}, \\
w_{, n}=0 & \text { on } S_{1}^{T}, \\
w=0 & \text { on } S_{2}^{T}, \\
\left.w\right|_{t=0}=w(0) & \text { in } \Omega .
\end{array}
$$

LEMma 2.7. Let $F_{3}, h$ and $v$ be given. Then $\chi$ is a solution to the problem

$$
\begin{aligned}
& \chi, t+v \cdot \nabla \chi-h_{3} \chi+h_{2} w_{x_{1}}-h_{1} w_{x_{2}}-\nu \Delta \chi=F_{3} \quad \text { in } \Omega^{T}, \\
& \chi=v_{i}\left(n_{i, x_{j}} \tau_{1 j}+\tau_{1 i, x_{j}} n_{j}\right)+v \cdot \tau_{1}\left(\tau_{12, x_{1}}-\tau_{11, x_{2}}\right) \equiv \chi_{*} \quad \text { on } S_{1}^{T} \text {, } \\
& \chi_{, x_{3}}=0 \quad \text { on } S_{2}^{T} \text {, } \\
& \left.\chi\right|_{t=0}=\chi(0) \quad \text { in } \Omega \text {. }
\end{aligned}
$$

For the detailed proofs of Lemmas 2.3-2.7 we refer the reader to [6].

3. Estimates. In this section we will present the estimates for $v$ and $h$ in the norms of $W_{2}^{2,1}\left(\Omega^{t}\right)$ and $W_{\sigma}^{2,1}\left(\Omega^{t}\right)$ respectively $(\sigma$ will be defined later, see Lemma 3.4) in terms of the initial and the external data and of the quantity $\|h\|_{L_{\infty}\left(0, t ; L_{3}(\Omega)\right)}$. These estimates are obtained on any time interval of the form $(k T,(k+1) T)$ by application of cut-off functions defined by

$$
\zeta^{\left(k_{n}\right)}(t)= \begin{cases}1 & \text { for } t \in((k-n) T,(k+1) T) \\ 0 & \text { for } t \leq(k-n-1) T\end{cases}
$$

where $\zeta^{\left(k_{n}\right)} \in \mathcal{C}_{0}^{\infty}(0, \infty)$ and $\dot{\zeta}^{\left(k_{n}\right)} \leq 1 / T$. It is easy to see that for fixed $k$ and increasing $n$ we have the inclusions $\operatorname{supp} \zeta^{\left(k_{0}\right)} \subset \operatorname{supp} \zeta^{\left(k_{1}\right)} \subset \cdots \subset$ $\operatorname{supp} \zeta^{\left(k_{n}\right)}$.

From now on we will use the notation $u^{\left(k_{n}\right)}=u \cdot \zeta^{k_{n}}$, where $0 \leq t \leq$ $(k+1) T$.

The first step is to estimate the third component of the vorticity field, which we denote by $\chi$. Since we integrate by parts, we expect the boundary integrals to vanish. Therefore we consider a function $\bar{\chi}$ defined as a solution 
to the problem

$$
\begin{array}{ll}
\bar{\chi}_{, t}-\nu \Delta \bar{\chi}=0 & \text { in } \Omega^{T}, \\
\bar{\chi}=\chi_{*} & \text { on } S_{1}^{T}, \\
\bar{\chi}_{, x_{3}}=0 & \text { on } S_{2}^{T}, \\
\left.\bar{\chi}\right|_{t=0}=0 & \text { in } \Omega
\end{array}
$$

and subtract it from the function $\chi$,

$$
\chi^{\prime}=\chi-\bar{\chi}
$$

Then $\chi^{\prime}$ is a solution to the problem

$$
\begin{array}{ll}
\chi_{, t}^{\prime}+v \cdot \nabla \chi^{\prime}-h_{3} \chi^{\prime}+h_{2} w_{, x_{1}}-h_{1} w_{, x_{2}}-\nu \Delta \chi^{\prime} & \\
\quad=F_{3}-v \nabla \bar{\chi}+h_{3} \bar{\chi} & \text { in } \Omega^{T}, \\
\chi^{\prime}=0 & \text { on } S_{1}^{T}, \\
\chi_{, x_{3}}^{\prime}=0 & \text { on } S_{2}^{T}, \\
\left.\chi^{\prime}\right|_{t=0}=\chi(0) & \text { in } \Omega .
\end{array}
$$

LEMmA 3.1. Let $h^{\left(k_{n}\right)} \in L_{\infty}\left(0, t ; L_{3}(\Omega)\right), v^{\prime\left(k_{n}\right)} \in L_{\infty}\left(0, t ; H^{5 / 6}(\Omega)\right)$, $F_{3}^{\left(k_{n}\right)} \in L_{2}\left(0, t ; L_{6 / 5}(\Omega)\right)$. Then a solution to the problem $(2.2)$ satisfies

$$
\begin{aligned}
& \left\|\chi^{\left(k_{n}\right)}\right\|_{V_{2}^{0}\left(\Omega^{t}\right)}^{2} \leq c d_{2}^{2}\left\|h^{\left(k_{n}\right)}\right\|_{L_{\infty}\left(0, t ; L_{3}(\Omega)\right)}^{2}+c\left\|F_{3}^{\left(k_{n}\right)}\right\|_{L_{2}\left(0, t ; L_{6 / 5}(\Omega)\right)}^{2} \\
& +c\left(d_{2}^{2}+1\right)\left\|v^{\prime\left(k_{n}\right)}\right\|_{L_{\infty}\left(0, t ; H^{5 / 6}(\Omega)\right)}+c\left\|v^{\prime\left(k_{n}\right)}\right\|_{W_{2}^{1,1 / 2}\left(\Omega^{t}\right)}+c d_{1}^{2}+2(n+2) d_{2}^{2} .
\end{aligned}
$$

Proof. Multiplying $(3.3)_{1}$ by $\zeta^{\left(k_{n}\right)}$, then by $\chi^{\prime\left(k_{n}\right)}$ and integrating over $\Omega$ and using the boundary conditions $(3.3)_{2},(3.3)_{3}$ and $(1.1)_{2}$ yields

$$
\begin{aligned}
\frac{1}{2} \frac{d}{d t}\left\|\chi^{\prime\left(k_{n}\right)}\right\|_{L_{2}(\Omega)}^{2}+\nu\left\|\nabla \chi^{\prime\left(k_{n}\right)}\right\|_{L_{2}(\Omega)}^{2} & =\int_{\Omega} h_{3}\left(\chi^{\prime\left(k_{n}\right)}\right)^{2} d x \\
& -\int_{\Omega}\left(h_{2} w_{, x_{1}}-h_{1} w_{, x_{2}}\right)^{\left(k_{n}\right)} \chi^{\prime\left(k_{n}\right)} d x+\int_{\Omega} F_{3}^{\left(k_{n}\right)} \chi^{\prime\left(k_{n}\right)} d x \\
& -\int_{\Omega}(v \cdot \nabla \bar{\chi})^{\left(k_{n}\right)} \chi^{\prime\left(k_{n}\right)} d x+\int_{\Omega} h_{3} \bar{\chi}^{\left(k_{n}\right)} \chi^{\prime\left(k_{n}\right)} d x+\dot{\zeta}^{\left(k_{n}\right)} \int_{\Omega} \chi^{\prime} \chi^{\prime\left(k_{n}\right)} d x .
\end{aligned}
$$

Now we estimate the terms on the right-hand side above. We have

$$
\begin{aligned}
\frac{d}{d t}\left\|\chi^{\prime\left(k_{n}\right)}\right\|_{L_{2}(\Omega)}^{2}+2 \bar{\nu}\left\|\chi^{\prime\left(k_{n}\right)}\right\|_{H^{1}(\Omega)}^{2} & \\
\leq & \varepsilon_{1}\left\|\chi^{\prime\left(k_{n}\right)}\right\|_{L_{6}(\Omega)}^{2}+\frac{1}{\varepsilon_{1}}\left\|h^{\left(k_{n}\right)}\right\|_{L_{3}(\Omega)}^{2}\left\|\chi^{\prime}\right\|_{L_{2}(\Omega)}^{2} \\
& +\varepsilon_{2}\left\|\chi^{\prime\left(k_{n}\right)}\right\|_{L_{6}(\Omega)}^{2}+\frac{1}{\varepsilon_{2}}\left\|h^{\left(k_{n}\right)}\right\|_{L_{3}(\Omega)}^{2}\|\nabla w\|_{L_{2}(\Omega)}^{2}
\end{aligned}
$$




$$
\begin{aligned}
& +\varepsilon_{3}\left\|\chi^{\prime\left(k_{n}\right)}\right\|_{L_{6}(\Omega)}^{2}+\frac{1}{\varepsilon_{3}}\left\|F_{3}^{\left(k_{n}\right)}\right\|_{L_{6 / 5}(\Omega)}^{2} \\
& +\varepsilon_{4}\left\|\nabla \chi^{\prime\left(k_{n}\right)}\right\|_{L_{2}(\Omega)}^{2}+\frac{1}{\varepsilon_{4}}\left\|\bar{\chi}^{\left(k_{n}\right)}\right\|_{L_{3}(\Omega)}^{2}\|v\|_{L_{6}(\Omega)}^{2} \\
& +\varepsilon_{5}\left\|\chi^{\prime\left(k_{n}\right)}\right\|_{L_{6}(\Omega)}^{2}+\frac{1}{\varepsilon_{5}}\|h\|_{L_{2}(\Omega)}^{2}\left\|\bar{\chi}^{\left(k_{n}\right)}\right\|_{L_{3}(\Omega)}^{2} \\
& +\varepsilon_{6}\left\|\chi^{\prime} \dot{\zeta}^{\left(k_{n}\right)}\right\|_{L_{2}(\Omega)}^{2}+\frac{1}{\varepsilon_{6}}\left\|\chi^{\prime\left(k_{n}\right)}\right\|_{L_{2}(\Omega)}^{2},
\end{aligned}
$$

where $\bar{\nu}=\nu / c$ and $c$ comes from the Poincaré inequality. Now we take $\varepsilon_{1}, \ldots, \varepsilon_{6}$ sufficiently small and use equality (3.2) and the Minkowski inequality for $\left\|\chi^{\prime} \dot{\zeta}^{\left(k_{n}\right)}\right\|_{L_{2}(\Omega)}^{2}$ to obtain

$$
\begin{aligned}
\frac{d}{d t}\left\|\chi^{\prime\left(k_{n}\right)}\right\|_{L_{2}(\Omega)}^{2}+\bar{\nu}\left\|\chi^{\prime\left(k_{n}\right)}\right\|_{H^{1}(\Omega)}^{2} \leq c\left\|h^{\left(k_{n}\right)}\right\|_{L_{3}(\Omega)}^{2}\left(\|\chi\|_{L_{2}(\Omega)}^{2}+\|\bar{\chi}\|_{L_{2}(\Omega)}^{2}\right) \\
+c\left\|h^{\left(k_{n}\right)}\right\|_{L_{3}(\Omega)}^{2}\|\nabla w\|_{L_{2}(\Omega)}^{2}+\left\|F_{3}^{\left(k_{n}\right)}\right\|_{L_{6 / 5}(\Omega)}^{2} \\
+c\left\|\bar{\chi}^{\left(k_{n}\right)}\right\|_{L_{3}(\Omega)}^{2}\|v\|_{L_{6}(\Omega)}^{2}+c\|h\|_{L_{2}(\Omega)}^{2}\left\|\bar{\chi}^{\left(k_{n}\right)}\right\|_{L_{3}(\Omega)}^{2} \\
+c\left(\left\|\chi \dot{\zeta}^{\left(k_{n}\right)}\right\|_{L_{2}(\Omega)}^{2}+\left\|\bar{\chi} \dot{\zeta}^{\left(k_{n}\right)}\right\|_{L_{2}(\Omega)}^{2}\right) .
\end{aligned}
$$

Integrating with respect to $t \in((k-n-1) T,(k+1) T)$ and using inequality (3.6) yields

$$
\begin{gathered}
\left\|\chi^{\prime\left(k_{n}\right)}(t)\right\|_{L_{2}(\Omega)}^{2}+\bar{\nu} \int_{(k-n-1) T}^{t}\left\|\chi^{\prime\left(k_{n}\right)}(s)\right\|_{H^{1}(\Omega)}^{2} d s \leq 2 c d_{2}^{2}\left\|h^{\left(k_{n}\right)}\right\|_{L_{\infty}\left(0, t ; L_{3}(\Omega)\right)}^{2} \\
\quad+c\left\|F_{3}^{\left(k_{n}\right)}\right\|_{L_{2}\left(0, t ; L_{6 / 5}(\Omega)\right)}^{2}+2 c d_{2}^{2}\left\|\bar{\chi}^{\left(k_{n}\right)}\right\|_{L_{\infty}\left(0, t ; L_{3}(\Omega)\right)}^{2}+2(n+2) d_{2}^{2},
\end{gathered}
$$

because from the definition of $\chi$ and $\chi^{\prime}$ it follows that

$$
\int_{(k-n-1) T}^{(k+1) T}\left(\left\|\chi \dot{\zeta}^{\left(k_{n}\right)}\right\|_{L_{2}(\Omega)}^{2}+\left\|\bar{\chi} \dot{\zeta}^{\left(k_{n}\right)}\right\|_{L_{2}(\Omega)}^{2}\right) d t<2(n+2) d_{2}^{2},
$$

where $d_{2}$ comes from Lemma 2.2. For a solution to (3.1) we have

$$
\left\|\bar{\chi}^{\left(k_{n}\right)}\right\|_{L_{\infty}\left(0, t ; L_{3}(\Omega)\right)} \leq c\left\|v^{\prime\left(k_{n}\right)}\right\|_{L_{\infty}\left(0, t ; H^{5 / 6}(\Omega)\right)},
$$

hence

$$
\begin{aligned}
\left\|\chi^{\prime\left(k_{n}\right)}\right\|_{V_{2}^{0}\left(\Omega^{t}\right)}^{2} \leq & c d_{2}^{2}\left\|h^{\left(k_{n}\right)}\right\|_{L_{\infty}\left(0, t ; L_{3}(\Omega)\right)}^{2}+c\left\|F_{3}^{\left(k_{n}\right)}\right\|_{L_{2}\left(0, t ; L_{6 / 5}(\Omega)\right)}^{2} \\
& +c d_{2}^{2}\left\|v^{\prime\left(k_{n}\right)}\right\|_{L_{\infty}\left(0, t ; H^{5 / 6}(\Omega)\right)}^{2}+2(n+2) d_{2}^{2}
\end{aligned}
$$


The trace theorem implies that

$$
\begin{aligned}
&\left\|\bar{\chi}^{\left(k_{n}\right)}\right\|_{L_{\infty}\left(0, t ; L_{2}(\Omega)\right)}^{2} \leq c\left\|v^{\left(k_{n}\right)}\right\|_{L_{\infty}\left(0, t ; H^{1 / 2+\varepsilon}(\Omega)\right)}^{2}, \\
&\left\|\bar{\chi}^{\left(k_{n}\right)}\right\|_{L_{2}\left(0, t ; H^{1}(\Omega)\right)}^{2} \leq c\left\|v^{\left(k_{n}\right)}\right\|_{W_{2}^{1,1 / 2}\left(\Omega^{t}\right)}^{2}
\end{aligned}
$$

Since

$$
\left\|v^{\prime\left(k_{n}\right)}\right\|_{L_{\infty}\left(0, t ; H^{1 / 2+\varepsilon}(\Omega)\right)}^{2} \leq c\left\|v^{\prime\left(k_{n}\right)}\right\|_{L_{\infty}\left(0, t ; H^{5 / 6}(\Omega)\right)}^{2}+c d_{1}^{2},
$$

the proof is finished.

We can finally find the estimate for $v$ in the $W_{2}^{2,1}(\Omega \times(k T,(k+1) T))$ norm (see Lemma 3.3). However, first we need an auxiliary inequality for $v^{\prime}=\left(v_{1}, v_{2}\right)$ in the $V_{2}^{1}\left(\Omega^{t}\right)$-norm (see Lemma 3.2). Consider the problem

$$
\begin{array}{ll}
v_{1, x_{2}}-v_{2, x_{1}}=\chi & \text { in } \Omega^{\prime}, \\
v_{1, x_{1}}+v_{2, x_{2}}=-h_{3} & \text { in } \Omega^{\prime}, \\
v^{\prime} \cdot \bar{n}^{\prime}=0 & \text { on } S_{1}^{\prime},
\end{array}
$$

where $\Omega^{\prime}=\Omega \cap\left\{x_{3}=\right.$ const $\left.\in(-a, a)\right\}$ and $S_{1}^{\prime}$ is defined analogously.

Lemma 3.2. Let $h^{\left(k_{n}\right)} \in L_{\infty}\left(0, t ; L_{3}(\Omega)\right), v^{\prime\left(k_{n}\right)} \in H^{1 / 2}\left(0, t ; L_{2}(\Omega)\right)$, $g^{\left(k_{n}\right)} \in L_{2}\left(\Omega^{t}\right), f_{3}^{\left(k_{n}\right)} \in L_{2}\left(S_{2}^{t}\right), F_{3}^{\left(k_{n}\right)} \in L_{2}\left(0, t ; L_{6 / 5}(\Omega)\right)$. Then

$$
\begin{aligned}
\left\|v^{\prime\left(k_{n}\right)}\right\|_{V_{2}^{1}\left(\Omega^{t}\right)}^{2} \leq & c d_{2}^{2}\left\|h^{\left(k_{n}\right)}\right\|_{L_{\infty}\left(0, t ; L_{3}(\Omega)\right)}^{2}+\left\|v^{\prime\left(k_{n}\right)}\right\|_{H^{1 / 2}\left(0, t ; L_{2}(\Omega)\right)}^{2} \\
& +c\left\|F_{3}^{\left(k_{n}\right)}\right\|_{L_{2}\left(0, t ; L_{6 / 5}(\Omega)\right)}^{2}+c\left\|f_{3}^{\left(k_{n}\right)}\right\|_{L_{2}\left(S_{2}^{t}\right)}^{2} \\
& +c\left\|g^{\left(k_{n}\right)}\right\|_{L_{2}\left(\Omega^{t}\right)}^{2}+c d_{1}^{2}+(c+3(n+2)) d_{2}^{2} .
\end{aligned}
$$

Proof. First we observe that applying the cut-off function $\zeta^{\left(k_{n}\right)}$ in the proof of Lemma 2.4 (see [6, Sec. 4, Lemma 4.2]) gives

$$
\begin{aligned}
\left\|h^{\left(k_{n}\right)}\right\|_{V_{2}^{0}\left(\Omega^{T}\right)}^{2} \leq & c d_{2}^{2}\left\|h^{\left(k_{n}\right)}\right\|_{L_{\infty}\left(0, t ; L_{3}(\Omega)\right)}^{2}+c\left\|f_{3}^{\left(k_{n}\right)}\right\|_{L_{2}\left(S_{2}^{t}\right)}^{2} \\
& +c\left\|g^{\left(k_{n}\right)}\right\|_{L_{2}\left(\Omega^{t}\right)}^{2}+(n+2) d_{2}^{2} .
\end{aligned}
$$

For a solution to the problem (3.5) we have the estimate

$$
\left\|v^{\prime\left(k_{n}\right)}\right\|_{V_{2}^{1}\left(\Omega^{t}\right)}^{2} \leq c\left\|h^{\left(k_{n}\right)}\right\|_{V_{2}^{0}\left(\Omega^{t}\right)}^{2}+c\left\|\chi^{\left(k_{n}\right)}\right\|_{V_{2}^{0}\left(\Omega^{t}\right)}^{2} .
$$

It follows by interpolation that

$$
\left\|v^{\prime\left(k_{n}\right)}\right\|_{L_{\infty}\left(0, t ; H^{5 / 6}(\Omega)\right)}^{2} \leq \varepsilon\left\|v^{\prime\left(k_{n}\right)}\right\|_{L_{\infty}\left(0, t ; H^{1}(\Omega)\right)}^{2}+c(1 / \varepsilon) d_{1}^{2}
$$

and from Lemma 2.2 that

$$
\left\|v^{\prime\left(k_{n}\right)}\right\|_{L_{2}\left(0, t ; H^{1}(\Omega)\right)}^{2} \leq c d_{2}^{2} .
$$

Applying Lemma 3.1, the inequalities (3.6) and (3.7) and using the above interpolation result ends the proof. 
Lemma 3.3. Let $k \in \mathbb{N} \backslash\{0,1\}$ be fixed and $k-n-1>0$. Assume that

$$
\begin{aligned}
\left(D^{\left(k_{n}\right)}\right)^{2}= & \left\|F_{3}^{\left(k_{n}\right)}\right\|_{L_{2}\left(0, t ; L_{6 / 5}(\Omega)\right)}^{2}+\left\|f_{3}^{\left(k_{n}\right)}\right\|_{L_{2}\left(S_{2}^{t}\right)}^{2} \\
& +\left\|g^{\left(k_{n}\right)}\right\|_{L_{2}\left(\Omega^{t}\right)}^{2}+c d_{1}^{2}+(c+3(n+2)) d_{2}^{2}<\infty
\end{aligned}
$$

and $\left\|h^{\left(k_{n+1}\right)}\right\|_{L_{\infty}\left(0, t ; L_{3}(\Omega)\right)},\left\|h^{\left(k_{n+1}\right)}\right\|_{L_{10 / 3}\left(\Omega^{t}\right)},\left\|f^{\left(k_{n+1}\right)}\right\|_{L_{2}\left(\Omega^{t}\right)}<\infty$. Then for any solution to the problem (1.1) we have the estimate

$$
\begin{aligned}
\left\|v^{\left(k_{n}\right)}\right\|_{W_{2}^{2,1}\left(\Omega^{t}\right)}+\left\|\nabla p^{\left(k_{n}\right)}\right\|_{L_{2}\left(\Omega^{t}\right)} \leq c d_{2}^{2}\left(\left\|h^{\left(k_{n+1}\right)}\right\|_{L_{\infty}\left(0, t ; L_{3}(\Omega)\right)}^{2}\right. \\
\left.+\left\|h^{\left(k_{n+1}\right)}\right\|_{L_{10 / 3}\left(\Omega^{t}\right)}^{2}+\left\|f^{\left(k_{n+1}\right)}\right\|_{L_{5 / 3}\left(\Omega^{t}\right)}^{2}\right) \\
+c\left(D^{\left(k_{n+1}\right)}\right)^{2}+c\left\|f^{\left(k_{n+1}\right)}\right\|_{L_{2}\left(\Omega^{t}\right)} .
\end{aligned}
$$

Proof. Let us consider the problem (1.1) in the form

$$
\begin{array}{ll}
v_{, t}^{\left(k_{n}\right)}-\operatorname{div} \mathbb{T}\left(v^{\left(k_{n}\right)}, p^{\left(k_{n}\right)}\right) & \\
\quad=-v^{\prime\left(k_{n}\right)} \cdot \nabla^{\prime} v-w h^{\left(k_{n}\right)}+f^{\left(k_{n}\right)}+\dot{\zeta}^{\left(k_{n}\right)} v & \text { in } \Omega^{t}, \\
\operatorname{div} v^{\left(k_{n}\right)}=0 & \text { in } \Omega^{t}, \\
v^{\left(k_{n}\right)} \cdot n=0 & \text { on } S^{t}, \\
n \cdot \mathbb{T}\left(v^{\left(k_{n}\right)}, p^{\left(k_{n}\right)}\right) \cdot \tau_{\alpha}=0, & \alpha=1,2, \\
\left.v^{\left(k_{n}\right)}\right|_{t=(k-n-1) T}=0 & \text { on } S^{t},
\end{array}
$$

where $v^{\prime}=\left(v_{1}, v_{2}\right), \nabla^{\prime}=\left(\partial_{x_{1}}, \partial_{x_{2}}\right)$. In view of [7, Lemma 3.7] the inequality $\left\|v^{\prime}\right\|_{L_{10}\left(\Omega^{t}\right)} \leq c\left\|v^{\prime}\right\|_{V_{2}^{1}\left(\Omega^{t}\right)}$ holds. Hence

$$
\begin{aligned}
\left\|v^{\prime\left(k_{n}\right)} \cdot \nabla^{\prime} v\right\|_{L_{5 / 3}\left(\Omega^{t}\right)} & \leq\left\|v^{\prime\left(k_{n}\right)}\right\|_{L_{10}\left(\Omega^{t}\right)}\left\|\nabla^{\prime} v\right\|_{L_{2}\left(\Omega^{t}\right)} \leq c d_{2}\left\|v^{\prime\left(k_{n}\right)}\right\|_{V_{2}^{1}\left(\Omega^{t}\right)}, \\
\left\|w h^{\left(k_{n}\right)}\right\|_{L_{5 / 3}\left(\Omega^{t}\right)} & \leq\|w\|_{L_{10 / 3}\left(\Omega^{t}\right)}\left\|h^{\left(k_{n}\right)}\right\|_{L_{10 / 3}\left(\Omega^{t}\right)} \leq c d_{2}\left\|h^{\left(k_{n}\right)}\right\|_{L_{10 / 3}\left(\Omega^{t}\right)} .
\end{aligned}
$$

In view of Lemma 3.2 we obtain, for any solution to the problem (3.9),

$$
\left\|v^{\left(k_{n}\right)}\right\|_{W_{5 / 3}^{2,1}\left(\Omega^{t}\right)} \leq c d_{2}\left(\left\|v^{\left(k_{n}\right)}\right\|_{V_{2}^{1}\left(\Omega^{t}\right)}+\left\|h^{\left(k_{n}\right)}\right\|_{L_{10 / 3}\left(\Omega^{t}\right)}\right)+c\left\|f^{\left(k_{n}\right)}\right\|_{L_{5 / 3}\left(\Omega^{t}\right)} .
$$

Applying now the interpolation result

$$
\left\|v^{\left(k_{n}\right)}\right\|_{H^{1 / 2}\left(0, t ; L_{2}(\Omega)\right)} \leq \varepsilon\left\|v^{\prime\left(k_{n}\right)}\right\|_{W_{5 / 3}^{2,1}\left(\Omega^{t}\right)}+c(1 / \varepsilon) d_{2}
$$

we get

$$
\begin{aligned}
\left\|v^{\left(k_{n}\right)}\right\|_{W_{5 / 3}^{2,1}\left(\Omega^{t}\right)} \leq & c d_{2}\left(\left\|h^{\left(k_{n}\right)}\right\|_{L_{\infty}\left(0, t ; L_{3}(\Omega)\right)}\right. \\
& \left.+\left\|h^{\left(k_{n}\right)}\right\|_{L_{10 / 3}\left(\Omega^{t}\right)}+D^{\left(k_{n}\right)}\right)+c\left\|f^{\left(k_{n}\right)}\right\|_{L_{5 / 3}\left(\Omega^{t}\right)} .
\end{aligned}
$$


Let us now rewrite problem (1.1) in the form

$$
\begin{array}{ll}
v_{, t}^{\left(k_{n}\right)}-\operatorname{div} \mathbb{T}\left(v^{\left(k_{n}\right)}, p^{\left(k_{n}\right)}\right) & \\
\quad=-v^{\prime} \cdot \nabla^{\prime} v^{\left(k_{n}\right)}-w^{\left(k_{n}\right)} h+f^{\left(k_{n}\right)}+\dot{\zeta}^{\left(k_{n}\right)} v & \text { in } \Omega^{t}, \\
\operatorname{div} v^{\left(k_{n}\right)}=0 & \text { in } \Omega^{t}, \\
v^{\left(k_{n}\right)} \cdot n=0 & \text { on } S^{t}, \\
n \cdot \mathbb{T}\left(v^{\left(k_{n}\right)}, p^{\left(k_{n}\right)}\right) \cdot \tau_{\alpha}=0, & \alpha=1,2, \\
\left.v^{\left(k_{n}\right)}\right|_{t=(k-n-1) T}=0 & \text { on } S^{t}, \\
\end{array}
$$

Then using (3.10) we get

$$
\begin{aligned}
\left\|v^{\prime} \cdot \nabla^{\prime} v^{\left(k_{n}\right)}\right\|_{L_{2}\left(\Omega^{t}\right)} \leq & \left\|v^{\left(k_{n+1}\right)}\right\|_{V_{2}^{1}\left(\Omega^{t}\right)}\left\|v^{\left(k_{n}\right)}\right\|_{W_{5 / 3}^{2,1}\left(\Omega^{t}\right)} \\
\leq & c d_{2}^{2}\left(\left\|h^{\left(k_{n+1}\right)}\right\|_{L_{\infty}\left(0, t ; L_{3}(\Omega)\right)}^{2}+\left\|h^{\left(k_{n+1}\right)}\right\|_{L_{10 / 3}\left(\Omega^{t}\right)}^{2}\right) \\
& +c\left(D^{\left(k_{n+1}\right)}\right)^{2}+c\left\|f^{\left(k_{n}\right)}\right\|_{L_{5 / 3}\left(\Omega^{t}\right)}^{2}
\end{aligned}
$$

and

$$
\begin{aligned}
\left\|w^{\left(k_{n}\right)} h\right\|_{L_{2}\left(\Omega^{t}\right)} \leq & \left\|w^{\left(k_{n}\right)}\right\|_{W_{5 / 3}^{2,1}\left(\Omega^{t}\right)}\left\|h^{\left(k_{n+1}\right)}\right\|_{L_{10 / 3}\left(\Omega^{t}\right)} \\
\leq & c d_{2}^{2}\left(\left\|h^{\left(k_{n+1}\right)}\right\|_{L_{\infty}\left(0, t ; L_{3}(\Omega)\right)}^{2}+\left\|h^{\left(k_{n+1}\right)}\right\|_{L_{10 / 3}\left(\Omega^{t}\right)}^{2}\right) \\
& +c\left(D^{\left(k_{n+1}\right)}\right)^{2}+c\left\|f^{\left(k_{n}\right)}\right\|_{L_{5 / 3}\left(\Omega^{t}\right)}^{2}
\end{aligned}
$$

which concludes the proof.

LEMMA 3.4. Let $\sigma \in(1,10)$ and assume that the norms $\left\|f^{\left(k_{n+1}\right)}\right\|_{L_{2}\left(\Omega^{t}\right)}$ and $\left\|g^{\left(k_{n}\right)}\right\|_{L_{\sigma}\left(\Omega^{t}\right)}$ are finite for any $k \in \mathbb{N} \backslash\{0,1\}$. Let $k-n-1>0$. Then for any solution to the problem (2.1) we have

$$
\begin{aligned}
\left\|h^{\left(k_{n}\right)}\right\|_{W_{\sigma}^{2,1}\left(\Omega^{t}\right)} & \\
\leq & \varphi\left(\|h\|_{W_{\sigma}^{2,1}(\Omega \times((k-n-4) T, k T))}+\left\|h^{\left(k_{0}\right)}\right\|_{W_{\sigma}^{2,1}\left(\Omega^{t}\right)}+D^{\left(k_{n+1}\right)}\right. \\
& +\left\|f^{\left(k_{n+1}\right)}\right\|_{\left.L_{2}\left(\Omega^{t}\right)\right) \delta_{k}(T)+c\left\|g^{\left(k_{n}\right)}\right\|_{L_{\sigma}\left(\Omega^{t}\right)}} \\
& +\frac{1}{T}\|h\|_{L_{\sigma}(\Omega \times((k-n-1) T,(k-n) T))},
\end{aligned}
$$

where $\varphi$ is some nonlinear, positive and increasing function. 
Proof. Let us consider problem (2.1) in the form

$$
\begin{array}{ll}
h_{, t}^{\left(k_{n}\right)}-\operatorname{div} \mathbb{T}\left(h^{\left(k_{n}\right)}, q^{\left(k_{n}\right)}\right) & \\
\quad=-v \cdot \nabla h^{\left(k_{n}\right)}-h^{\left(k_{n}\right)} \cdot \nabla v+g^{\left(k_{n}\right)}+\dot{\zeta}^{\left(k_{n}\right)} h & \text { in } \Omega^{t}, \\
\operatorname{div} h^{\left(k_{n}\right)}=0 & \text { in } \Omega^{t}, \\
h^{\left(k_{n}\right)} \cdot n=0, \quad n \cdot \mathbb{T}\left(h^{\left(k_{n}\right)}, q^{\left(k_{n}\right)}\right) \cdot \tau_{\alpha}=0, \quad \alpha=1,2, & \text { on } S_{1}^{t}, \\
h_{i}^{\left(k_{n}\right)}=0, \quad i=1,2, \quad h_{3}^{\left(k_{n}\right)}=0 & \text { on } S_{2}^{t}, \\
\left.h^{\left(k_{n}\right)}\right|_{t=(k-n-1) T}=0 & \text { in } \Omega .
\end{array}
$$

Repeating the proof of [4, Lemma 3.4] we obtain the inequality

$$
\begin{aligned}
\left\|h^{\left(k_{n}\right)}\right\|_{W_{\sigma}^{2,1}\left(\Omega^{t}\right)}+\left\|\nabla q^{\left(k_{n}\right)}\right\|_{L_{\sigma}\left(\Omega^{t}\right)} \leq \varphi\left(\left\|v^{\left(k_{n+1}\right)}\right\|_{W_{2}^{2,1}\left(\Omega^{t}\right)}\right)\left\|h^{\left(k_{n}\right)}\right\|_{L_{2}\left(\Omega^{t}\right)} \\
+c\left\|g^{\left(k_{n}\right)}\right\|_{L_{\sigma}\left(\Omega^{t}\right)}+\frac{1}{T}\|h\|_{L_{\sigma}(\Omega \times((k-n-1) T,(k-n) T))},
\end{aligned}
$$

for any $\sigma \in(1,10)$ and $\varphi$ some nonlinear, positive and increasing function. Next we estimate $\left\|h^{\left(k_{n}\right)}\right\|_{L_{2}\left(\Omega^{t}\right)}$. Therefore we multiply $(3.13)_{1}$ by $h^{\left(k_{n}\right)}$, integrate by parts over $\Omega$ and repeat the proof of Lemma 2.5 (for details, see [6, Sec. 4, Lemma 4.2]). Finally, we get

$$
\begin{aligned}
\left\|h^{\left(k_{n}\right)}\right\|_{L_{2}\left(\Omega^{t}\right)} \leq c \sqrt{n+2}\left(\left\|\nabla v^{\left(k_{n+2}\right)}\right\|_{L_{2}\left(0, t ; L_{3}(\Omega)\right)}\right. & \\
\cdot & \left.\exp \left(c\left\|\nabla v^{\left(k_{n+2}\right)}\right\|_{L_{2}\left(0, t ; L_{3}(\Omega)\right)}^{2}\right)+1\right) \delta_{k}(T) .
\end{aligned}
$$

Next we estimate the right-hand side using Lemma 3.3. Observing that

$$
\left\|h^{\left(k_{n+2}\right)}\right\|_{W_{\sigma}^{2,1}\left(\Omega^{t}\right)} \leq\|h\|_{W_{\sigma}^{2,1}(\Omega \times((k-n-4) T, k T))}+\left\|h^{\left(k_{0}\right)}\right\|_{W_{\sigma}^{2,1}\left(\Omega^{t}\right)}
$$

we conclude the proof.

Proof of Theorem 1. Let $T_{0}=4 T$, so $k=4$ and $n=0$ (when $n=0$ we write $k$ instead of $k_{0}$ ). In view of [4, Lemma 3.5],

$$
\|h\|_{L_{\sigma}(\Omega \times(3 T, 4 T))} \leq\left\|h^{(3)}\right\|_{W_{\sigma}^{2,1}\left(\Omega^{t}\right)} \leq\|h\|_{W_{\sigma}^{2,1}\left(\Omega \times\left(0, T_{0}\right)\right)} \leq A,
$$

where the constant $A$ is such that

$$
c\left(\|g\|_{L_{\sigma}\left(\Omega \times\left(0, T_{0}\right)\right)}+\|h(0)\|_{W_{\sigma}^{2-2 / \sigma}(\Omega)}\right)<A
$$

and the constant $c$ depends on $n, p$ and $T$ (for details see [4, Lemma 3.5]). Let us observe that without loss of generality we can assume that

$$
c\|g\|_{L_{\sigma}(\Omega \times(k T,(k+1) T))}<A
$$

for any $k \in \mathbb{N}$. Then (3.12) implies that

$$
\begin{aligned}
\left\|h^{(4)}\right\|_{W_{\sigma}^{2,1}\left(\Omega^{t}\right)} \leq & \varphi\left(A+\left\|h^{(4)}\right\|_{W_{\sigma}^{2,1}\left(\Omega^{t}\right)}+D^{\left(4_{1}\right)}+\left\|f^{\left(4_{1}\right)}\right\|_{L_{2}\left(\Omega^{t}\right)}\right) \delta_{4}(T) \\
& +c\left\|g^{(4)}\right\|_{L_{\sigma}\left(\Omega^{t}\right)}+\frac{1}{T}\|h\|_{L_{\sigma}(\Omega \times(3 T, 4 T))} .
\end{aligned}
$$


In view of (3.14) and for $\delta_{4}(T)$ sufficiently small,

$$
\begin{aligned}
\varphi\left(A+\left\|h^{(4)}\right\|_{W_{\sigma}^{2,1}\left(\Omega^{t}\right)}+D^{\left(4_{1}\right)}+\left\|f^{\left(4_{1}\right)}\right\|_{L_{2}\left(\Omega^{t}\right)}\right) & \delta_{4}(T) \\
& +c\left\|g^{(4)}\right\|_{L_{\sigma}\left(\Omega^{t}\right)}+\frac{A}{T} \leq A
\end{aligned}
$$

where

$$
c\left\|g^{(4)}\right\|_{L_{\sigma}\left(\Omega^{t}\right)}+\frac{A}{T}<A,
$$

if only $T$ is large enough. Hence

$$
\left\|h^{(4)}\right\|_{W_{\sigma}^{2,1}\left(\Omega^{t}\right)} \leq A .
$$

Assume now that for $n=0$ and $4 \leq m \leq s \in \mathbb{N}$ we have

$$
\left\|h^{(m)}\right\|_{W_{\sigma}^{2,1}\left(\Omega^{t}\right)} \leq A \text {. }
$$

We will show that

$$
\left\|h^{(s+1)}\right\|_{W_{\sigma}^{2,1}\left(\Omega^{t}\right)} \leq A .
$$

From (3.12) it follows that

$$
\begin{aligned}
\left\|h^{(s+1)}\right\|_{W_{\sigma}^{2,1}\left(\Omega^{t}\right)} & \\
\leq & \varphi\left(\left\|h^{(s-2)}\right\|_{W_{\sigma}^{2,1}\left(\Omega^{t}\right)}+\left\|h^{(s-1)}\right\|_{W_{\sigma}^{2,1}\left(\Omega^{t}\right)}+\left\|h^{(s)}\right\|_{W_{\sigma}^{2,1}\left(\Omega^{t}\right)}\right. \\
& \left.+\left\|h^{(s+1)}\right\|_{W_{\sigma}^{2,1}\left(\Omega^{t}\right)}+D^{(s+1)_{1}}+\left\|f^{(s+1)_{1}}\right\|_{L_{2}\left(\Omega^{t}\right)}\right) \delta_{s+1}(T) \\
& +c\left\|g^{(s+1)}\right\|_{L_{\sigma}\left(\Omega^{t}\right)}+\frac{1}{T}\|h\|_{L_{\sigma}(\Omega \times(s T,(s+1) T))} \\
\leq & \varphi\left(3 A+\left\|h^{(s+1)}\right\|_{W_{\sigma}^{2,1}\left(\Omega^{t}\right)}+D^{(s+1)_{1}}+\left\|f^{(s+1)_{1}}\right\|_{\left.L_{2}\left(\Omega^{t}\right)\right)} \delta_{s+1}(T)\right. \\
& +c\left\|g^{(s+1)}\right\|_{L_{\sigma}\left(\Omega^{t}\right)}+\frac{1}{T}\|h\|_{L_{\sigma}(\Omega \times(s T,(s+1) T))} .
\end{aligned}
$$

If $\delta_{s+1}(T)$ is small enough, then using (3.17) to estimate the last term on the right-hand side we can see that (3.18) holds for $T$ sufficiently large. The existence of functions $v$ and $h$ can be proved as in [4, Sec. 4]. This concludes the proof.

Proof of Theorem 2. It remains to show that $\|h(k T)\|_{L_{2}(\Omega)}$ is equally small for any $k \in \mathbb{N}$. We first differentiate (1.1) with respect to $x_{3}$, then multiply by $h$, integrate over $\Omega$, use the boundary conditions and apply the Stokes theorem and the Korn, Hölder and Young inequalities. Then we get

$$
\frac{d}{d t}\|h\|_{L_{2}(\Omega)}^{2}+\nu\|h\|_{H^{1}(\Omega)}^{2} \leq c\|h\|_{L_{2}(\Omega)}^{2}\|\nabla v\|_{L_{3}(\Omega)}^{2}+c\|g\|_{L_{2}(\Omega)}^{2}+c\left\|f_{3}\right\|_{L_{2}\left(S_{2}\right)}^{2} .
$$


Using the Gronwall inequality on the time interval $(k T,(k+1) T)$ yields

$$
\begin{aligned}
\| h((k+1) T) & \|_{L_{2}(\Omega)}^{2} \leq c e^{-\bar{\nu} T+c\|\nabla v\|_{L_{2}\left(k T,(k+1) T ; L_{3}(\Omega)\right)}^{2}} \\
& \cdot\left(\|h(k T)\|_{L_{2}(\Omega)}^{2}+\|g\|_{L_{2}(\Omega \times(k T,(k+1) T))}^{2}+\left\|f_{3}\right\|_{L_{2}\left(S_{2} \times(k T,(k+1) T)\right)}^{2}\right) .
\end{aligned}
$$

From Theorem 1 it follows that $\|\nabla v\|_{L_{2}\left(k T,(k+1) T ; L_{3}(\Omega)\right)}^{2}<c\left(A^{4}+1\right)$. Since the constant $A$ is chosen in such a way that it satisfies (3.15) and (3.16) we can take $T$ large enough so that $-\bar{\nu} T+c\left(A^{4}+1\right)<0$ and

$$
\begin{aligned}
c e^{-\bar{\nu} T+c\|\nabla v\|_{L_{2}\left(k T,(k+1) T ; L_{3}(\Omega)\right)}^{2}} & \\
\cdot\left(\|h(k T)\|_{L_{2}(\Omega)}^{2}+\|g\|_{L_{2}(\Omega \times(k T,(k+1) T))}^{2}\right. & \left.\left\|f_{3}\right\|_{L_{2}\left(S_{2} \times(k T,(k+1) T)\right)}^{2}\right) \\
& \leq c e^{-\bar{\nu} T+c\left(A^{4}+1\right)} \cdot 2 \varepsilon<\varepsilon .
\end{aligned}
$$

Applying Theorem 1 on any time interval $(k T,(k+1) T)$ ends the proof.

Proof of Theorem 3. Let $\left(v_{i}, p_{i}\right)$ for $i=1,2$ be two solutions to the problem (1.1). Let $V=v_{1}-v_{2}$ and $P=p_{1}-p_{2}$. Then the pair $\left(V^{\left(k_{n}\right)}, P^{\left(k_{n}\right)}\right)$ solves the problem

$$
\begin{array}{ll}
V_{, t}^{\left(k_{n}\right)}-\operatorname{div} \mathbb{T}\left(V^{\left(k_{n}\right)}, P^{\left(k_{n}\right)}\right)=-V^{\left(k_{n}\right)} \cdot \nabla v_{1}-v_{2} \cdot \nabla V^{\left(k_{n}\right)}+\dot{\zeta} V & \text { in } \Omega^{t}, \\
\operatorname{div} V^{\left(k_{n}\right)}=0 & \text { in } \Omega^{T}, \\
V^{\left(k_{n}\right)} \cdot n=0 & \text { on } S^{T}, \\
n \cdot \mathbb{T}\left(V^{\left(k_{n}\right)}, P^{\left(k_{n}\right)}\right) \cdot \tau_{\alpha}=0, \quad \alpha=1,2, & \text { on } S^{T}, \\
\left.V^{\left(k_{n}\right)}\right|_{t=k-n-1}=0 & \text { in } \Omega .
\end{array}
$$

Multiplying the first equation by $V^{\left(k_{n}\right)}$ and integrating over $\Omega$ gives

$$
\frac{d}{d t}\left\|V^{\left(k_{n}\right)}\right\|_{L_{2}(\Omega)}^{2}+\nu\left\|V^{\left(k_{n}\right)}\right\|_{H^{1}(\Omega)}^{2} \leq c\left\|\nabla v_{1}^{\left(k_{n+1}\right)}\right\|_{L_{3}(\Omega)}^{2}\left\|V^{\left(k_{n}\right)}\right\|_{L_{2}(\Omega)}^{2} .
$$

Since $v_{1}^{\left(k_{n+1}\right)} \in L_{\infty}\left((k-n-2) T,(k+1) T ; W_{3}^{1}(\Omega)\right)$, the Gronwall inequality implies that $\left\|V^{\left(k_{n}\right)}(t)\right\|_{L_{2}(\Omega)}=0$. This ends the proof.

Acknowledgements. Research of W. M. Zajączkowski is partially supported by MNiSW Grant No 1 PO3A 02130 and EC FP6 Marie Curie ToK Programme SPADE2.

\section{References}

[1] O. V. Besov, V. P. Il'in and S. M. Nikol'skiı̌, Integral Representations of Functions and Imbedding Theorems, Nauka, Moscow, 1975 (in Russian).

[2] P. B. Mucha, Stability of nontrivial solutions of the Navier-Stokes system on the three-dimensional torus, J. Differential Equations 172 (2001), 359-375. 
[3] B. Nowakowski and W. M. Zajączkowski, Global attractor for Navier-Stokes equations in cylindrical domains, Appl. Math. (Warsaw) 36 (2009), 183-194.

[4] J. Rencławowicz and W. M. Zajacczkowski, Large time regular solutions to the NavierStokes equations in cylindrical domains, Topol. Methods Nonlinear Anal. 32 (2008), 69-87.

[5] M. Wiegner and W. M. Zajączkowski, On stability of axially symmetric solutions to Navier-Stokes equations in a cylindrical domain and with boundary slip conditions, in: Banach Center Publ. 70, Inst. Math., Polish Acad. Sci., 2005, 251-278.

[6] W. M. Zajączkowski, Long time existence of regular solutions to Navier-Stokes equations in cylindrical domains under boundary slip conditions, Studia Math. 169 (2005), 243-285.

[7] —, Global special regular solutions to the Navier-Stokes equations in a cylindrical domain without the axis of symmetry, Topol. Methods Nonlinear Anal. 24 (2004), 69-105.

Bernard Nowakowski

Institute of Mathematics

Polish Academy of Sciences

Śniadeckich 8

00-956 Warszawa, Poland

E-mail: bernard@impan.pl
Wojciech M. Zajaczkowski

Institute of Mathematics

Polish Academy of Sciences

Sniadeckich 8

00-956 Warszawa, Poland

E-mail: wz@impan.pl

and

Institute of Mathematics and Cryptology

Military University of Technology

Kaliskiego 2

00-908 Warszawa, Poland

Received on 27.6.2007;

revised version on 23.1.2008 